\title{
IMPRESSIONS ABOUT THE COVID-19 PANDEMIC AND BONE MARROW TRANSPLANTATION
}

\section{OPINION LETTER}

\author{
Eduardo J. A. Paton \\ ONCOBIO and BIOCOR - Belo Horizonte/Minas Gerais
}

Correspondence to: dupaton@uol.com.br

Since the start of Covid-19 epidemic first cases in Belo Horizonte, Minas Gerais state capital (sixth largest city, second state with more inhabitants and third richest state in Brazil) we adopted several contingency measures at our Cell Therapy Unit in our Cancer Center. Our center has two infirmaries, both with HEPA filters, positive pressure rooms and isolation structures: the first one, with nine beds, for patients that need agressive chemotherapy treatments, like AML, ALL and relapsed lymphomas patients, and a second unit, with thirteen beds, for patients submited to hematopoietic cell transplants. We decided to keep the HCT unit as a "Covid-19 free zone", performing nasal and throat RT-PCR for SARS-Cov-2 two days before patients and familial care givers hospitalization at the unit, abolish visitation and familial care givers circulation during the hospitalization and performed weekly RT-PCR from the whole unit assistance team. The other unit was classified as a "yellow zone" with its own team and HEPA filter and positive pressure turned off to allow hospitalization of non tested patients or even Covid-19 positive patients in need of agressive treatment in order to decrease other patients and assistance team contamination risk.

Since april we had 35 medical assistance health workers put in two weeks quarantine due to respiratory symptoms with only four diagnosed with Covid-19, all of them dispensed until negativation of RT-PCR, none of them needed hospitalization due to the infection. No patients had documented infection by any of these workers. We had one 31 years old high risk ALL female patient who was diagnosed with Covid-19 the day before hospitalization for a HLA identical sibling HCT transplant which was postponed. She had a benign outcome of the viral infection but presented a hematologic and CNS relapse during her quarentine time, which was treated with high dose Methotrexate based regimen and reached complete CNS and bone marrow remission with a negative MRD. She is now on day +7 post an allogeneic MAC TBI based transplant, evolving with a mild hepatic VOD in regression with no other complications. No other programed transplants or chemotherapies were postponed due to the pandemic. No other patient developed Covid-19 since the start of contigency measures.

We believe that the contigency measures adopted sooner in the pandemic outcome was pivotal to keep our units open and allowed us to treat properly and in right time all the patients in need.

1.Recommendations of Sociedade Brasileira de Transplante de Medula Óssea (SBTMO)

2.Recommendations of American Society for Transplantation and Cellular Therapy (ASTCT)

3.Recommendations of European Bone Marrow Transplantion (EBMT) 